\title{
A NUMERICAL METHOD FOR DETERMINATION OF MOISTURE TRANSFER COEFFICIENT ACCORDING TO THE DIFFUSION MOISTURE PROFILES ${ }^{1}$
}

\author{
E. Pavlušováa ${ }^{a}$ M. Pavluš ${ }^{a, 2}$, I. Sarhadov ${ }^{b}$, \\ I. V. Amirkhanov ${ }^{c}$, T. P. Puzynina ${ }^{c}$, I. V. Puzynin ${ }^{c}$ \\ ${ }^{a}$ Technical University of Košice, Slovakia \\ ${ }^{b}$ Dushanbe University, Tadjikistan \\ ${ }^{c}$ Joint Institute for Nuclear Research, Dubna
}

For a set of the measured diffusion moisture profiles a numerical method for determination of the moisture transfer coefficient $D(w, t)$ is suggested. The transfer coefficient is found as a sum of the degree $p_{0} w^{p(t)}$ and exponential $A \mathrm{e}^{\mu\left(w-v_{0}\right)}$ functions of the moisture concentration $w$, as opposed to the previous works. The exponent $p(t)$ of the power function depends on the time $t$. The exponential function describes profiles for large times nearby the boundary of the sample, where evaporation of the moisture to the atmosphere takes place. A conservative difference scheme for numerical solution of the direct problem is suggested. An inverse problem for minimization of an error functional is solved by the Newton method. Thus, a more accurate coincidence of the calculated profiles of the moisture concentration with the measured profiles is gained.

Предложен численный метод определения коэффициента переноса влаги $D(w, t)$ для набора измеряемых диффузионных профилей влажности. Коэффициент переноса находится как сумма степенной $p_{0} w^{p(t)}$ и экспоненциальной $A \mathrm{e}^{\mu\left(w-v_{0}\right)}$ функций концентрации влаги $w$ в отличие от предыдущих работ. Экспонента $p(t)$ степенной функции зависит от времени $t$. Экспоненциальная функция описывает профили влаги для больших времен вблизи границы образца, где происходит испарение влаги в атмосферу. Предложена консервативная разностная схема для численного решения прямой задачи. Обратная задача минимизации функционала погрешности решается методом Ньютона. Следовательно, обеспечивается более точное совпадение вычисляемых профилей концентрации влаги с измеряемыми профилями.

PACS: 02.60.Cb; 02.90.+p

\section{INTRODUCTION}

A method of the moisture quantity analysis in building materials was suggested in [1]. The method is based on the exposure of a sample of a building material to neutrons that do not destroy the sample and registration of the signals of non-absorbed neutrons by the sample.

\footnotetext{
${ }^{1}$ Supported by grants RFBR No.06-01-00228, 05-01-00645-a and VEGA 1/1006/04, 1/1136/04, 1/2562/05, $1 / 2647 / 05$.

${ }^{2}$ Corresponding author.
} 
The experimental data of moisture distribution are presented as an example in a rectangular porous sample of size $3 \times 9 \times 12 \mathrm{~cm}$ during the drying of the sample. All sides of surfaces are water- and air-proof except one side with dimensions $3 \times 12 \mathrm{~cm}$ where evaporation of moisture to the atmosphere takes place. The moisture transfer coefficient is calculated on the assumption that one-dimensional moisture moves along the sample's width of the $9 \mathrm{~cm}$ size and is empirically determined in the form of a polynomial of the third order of the moisture concentration. The polynomial coefficients were calculated under a condition of a minimal quadratic functional that determines a diversion of theoretical results obtained by a numerical solution of the nonlinear diffusion equation, from the experimental data obtained in [1] (see Fig. 1,a). Later the solution was obtained in [2], too.
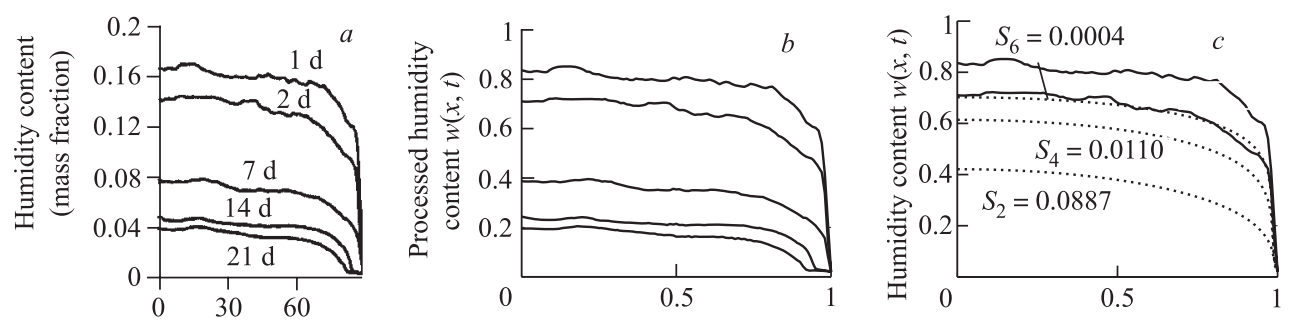

Fig. 1. a) Moisture distribution in dimensional units. Space variable $x$ in millimeters $(0,90)$ and moisture values $w$ in grams per $\mathrm{cm}^{3}(0 ; 0.2)$. Moisture profiles were obtained by neutron radiography method and by processing of appropriate neutron signals [1]. b) Moisture profiles in dimensionless units. Transformation of space variable $x=x / 9 \mathrm{~cm}$ and moisture values $w=w / 0.2 \mathrm{~g} / \mathrm{cm}^{3}$. c) Computed profiles (dotted lines) which approximate measured profile of the second day. The profiles are calculated for parameter $p_{0}=60$, different constant exponents $p=2, p=4, p=6$ and are denoted by corresponding functional values $S_{p}$

In this paper we deal with drying of a sample of porous material which is described in [1] and we construct a method for determination of a new type of moisture transfer coefficient. At the beginning we divide the abscissa axis interval $(0,90)$ of Fig. $1, a$ into 101 equidistant points and we display values $w$ of humidity content in these points in the dimensionless units in Fig. 1, b. Later, we compare the calculated moisture profiles just with the processed moisture profiles of Fig. 1, $b$. Notice that we consider the values of the first day of measuring $\varphi(x)$ as an initial condition in the next formulation of a direct problem.

\section{FORMULATION AND NUMERICAL SOLUTION OF DIRECT PROBLEM}

We consider the following diffusion problem of moisture transfer from the sample of porous material to the atmosphere:

$$
\frac{\partial w}{\partial t}=\frac{\partial}{\partial x}\left[D(w, t) \frac{\partial w}{\partial x}\right], \quad 0<x<1, \quad 0<t<1
$$

with initial

$$
w(x, 0)=\varphi(x), \quad 0<x<1
$$


and boundary conditions

$$
\begin{aligned}
\frac{\partial w}{\partial x}(0, t) & =0, \quad 0<t<1, \\
-D(w(1, t), t) \frac{\partial w}{\partial x}(1, t) & =1500\left[w(1, t)-v_{0}\right], \quad 0<t<1,
\end{aligned}
$$

where $v_{0}=0.019545$ is the smallest value in Fig. $1, b$. First, we introduce an equidistant net with time step $\tau$ and space step $h$

$$
\left\{t_{j}=j \tau,(j=0,1, \ldots, m) ; x_{i}=i h,(i=0,1, \ldots, n), h=1 / n\right\} .
$$

Then, the problem (1)-(4) can be approximated by the linearized difference scheme (5)-(8)

$$
\begin{gathered}
-C\left(w_{i-1}^{j+1(s)}\right) w_{i-1}^{j+1(s+1)}+\left[\bar{C}_{i}^{j+1(s)}+\frac{h^{2}}{\tau}\right] w_{i}^{j+1(s+1)}-C\left(w_{i}^{j+1(s)}\right) w_{i+1}^{j+1(s+1)}=0, \\
\bar{C}_{i}^{j+1(s)}=C\left(w_{i-1}^{j+1(s)}\right)+C\left(w_{i}^{j+1(s)}\right), \\
w_{i}^{0}=\varphi\left(x_{i}\right), \\
3 w_{0}^{j+1(s+1)}-4 w_{1}^{j+1(s+1)}+w_{2}^{j+1(s+1)}=0, \\
-D\left(w_{n}^{j+1(s)}, t_{j}\right) \frac{w_{n}^{j+1(s+1)}-w_{n-1}^{j+1(s+1)}}{h}=1500\left(w_{n}^{j+1(s+1)}-v_{0}\right),
\end{gathered}
$$

where $w_{i}^{j+1(s+1)}$ is a successive approximation to the approximation $w_{i}^{j+1(s)}$ for the $j+1$ time layer. The values $w_{i}^{j+1(s+1)}$ can be found by the Gauss elimination method. The scheme (5)-(8) is conservative and its order of approximation is $O\left(\tau+h^{2}\right)$.

\section{INITIAL DETERMINATION OF TRANSFER COEFFICIENT $D(w, t)$}

In general, we look for the transfer coefficient in the form

$$
D(w, t)=p_{0} w^{p}+1.5 \exp \left[-150\left(w-v_{0}\right)\right], \quad p=p\left(t, p_{1}, p_{2}, p_{3}\right), \quad 0 \leqslant t \leqslant 1,
$$

where the exponent $p$ is a function not only of $t$ but also of parameters $p_{1}, p_{2}, p_{3}$. We find out the set of parameters $D=\left[\begin{array}{llll}p_{0} & p_{1} & p_{2} & p_{3}\end{array}\right]$ by the Newton method so that the error functional

$$
S(D)=\sum_{j=1}^{M} \int_{0}^{1}\left|w\left(x, t_{j}\right)-w_{e}\left(x, t_{j}\right)\right|^{2} d x
$$

has a minimal value ( $M=5$ - number of days of measuring), where $w_{e}\left(x, t_{j}\right)$ are measured values of moisture profiles (see Fig. $1, b)$ and $w\left(x, t_{j}\right)$ are computed moisture profiles for given set of parameters $D$. The term $1.5 \exp \left[-150\left(w-v_{0}\right)\right]$ describes the behavior of calculated moisture profiles only for $w-v_{0} \sim 0+$ and $x \sim 1-$ because if, for example, $w-v_{0} \geqslant 0.1$ then the term $1.5 \exp \left[-150\left(w-v_{0}\right)\right] \cong 0$. Constants 1.5 and 150 are obtained by means of numerical solution of difference scheme (5)-(8). Hence, in the case $w-v_{0} \geqslant 0.1$, the term 
$p_{0} w^{p}$ plays the main role in the transfer coefficient $D(w, t)$. So, it is important to determine the exponent $p$ in (9).

Let us use fixed constants $p=2, p=4$ and $p=6$ and solve direct problem (1)-(4) only for $t$ : $0 \leqslant t \leqslant 1 / 20$, where the initial condition corresponds to the first day of measuring (see Fig. 1,c). After we solve the direct problem (1)-(4) for fixed constants $p=5 / 4, p=5 / 2, p=15 / 4$ and for $t: 1 / 20 \leqslant t \leqslant 6 / 20$, where the initial condition corresponds to the profile of the second day of measuring (see Fig. 2,a). Solving the direct problem (1)-(4) for $p=9 / 10, p=9 / 5, p=27 / 10$ and for $t$ : $6 / 20 \leqslant t \leqslant 13 / 20$, where the initial condition corresponds to the profile of the seventh day of measuring, we obtain
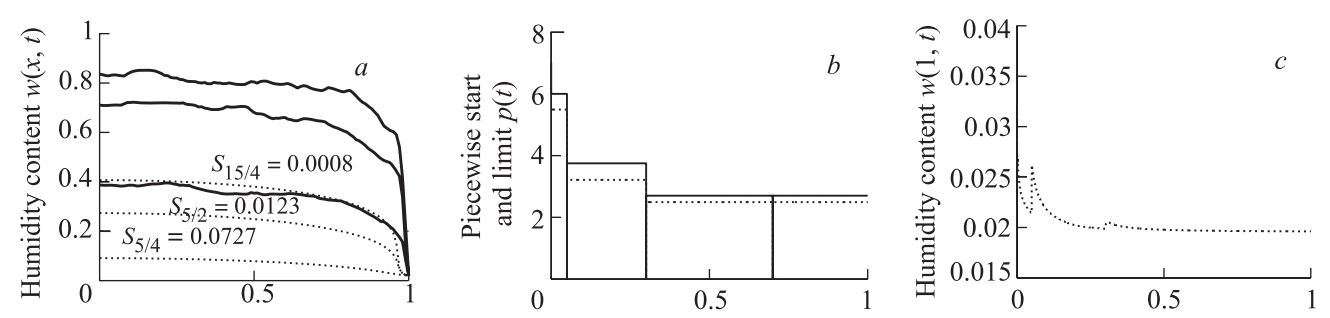

Fig. 2. a) Computed profiles (dotted lines) which approximate the seventh day of measuring. The profiles are calculated for parameter $p_{0}=60$, different constant exponents $p=5 / 4, p=5 / 2, p=15 / 4$ and are denoted by corresponding functional values $S_{p}$. b) Starting piecewise constant exponent $p(t)$ (solid line) and limiting piecewise constant exponent $p(t)$ (dotted line). $c$ ) Discontinuity of concentration $w(1, t)$ in the points $t=1 / 20$ and $t=6 / 20$

the corresponding functional values $S_{9 / 10}=0.0318, S_{9 / 5}=0.0085$ and $S_{27 / 10}=0.0001$. Finally, solving the direct problem (1)-(4) for the same $p=9 / 10, p=9 / 5, p=27 / 10$ and for $t: 13 / 20 \leqslant t \leqslant 1$, where the initial condition corresponds to the profile of the fourteenth day of measuring, we obtain the corresponding functional values $S_{9 / 10}=0.01884$, $S_{9 / 5}=0.00394$ and $S_{27 / 10}=0.00005$. Thus, solving numerically the set of four initial boundary value problems (1)-(4) with different initial conditions, we obtain an initial transfer coefficient (9), where exponent $p$ depends on time $t$ as follows:

$$
p(t)= \begin{cases}p_{1}, & 0 \leqslant t<1 / 20 \\ p_{2}, & 1 / 20 \leqslant t<6 / 20 \\ p_{3}, & 6 / 20 \leqslant t \leqslant 1\end{cases}
$$

and $p_{1}=6, p_{2}=15 / 4, p_{3}=27 / 10$.

\section{NEWTON METHOD AND CALCULATION RESULTS}

Let us consider the widely used Newton formula for minimization of functional (10) with respect to parameters $D=\left(p_{0}, p_{1}, p_{2}, p_{3}\right)$

$$
\begin{aligned}
& D^{(k+1)}=D^{(k)}-\left[S^{\prime \prime}\left(D^{(k)}\right)\right]^{-1} S^{\prime}\left(D^{(k)}\right), \quad D^{(0)}=\left[\begin{array}{llll}
p_{0}^{(0)} & p_{1}^{(0)} & p_{2}^{(0)} & p_{3}^{(0)}
\end{array}\right]^{T}, \\
& S^{\prime \prime}\left(D^{(k)}\right)=\left[\frac{\partial^{2} S}{\partial p_{r} \partial p_{s}}\right]_{r, s=0,1,2,3}, \quad S^{\prime}\left(D^{(k)}\right)=\left[\frac{\partial S}{\partial p_{0}} \frac{\partial S}{\partial p_{1}} \frac{\partial S}{\partial p_{2}} \frac{\partial S}{\partial p_{3}}\right]^{T}
\end{aligned}
$$


where $D^{(0)}$ is an initial (starting) iteration; $S^{\prime \prime}\left(D^{(k)}\right)$ is the symmetrical Hessian matrix; $S^{\prime}\left(D^{(k)}\right)$ is the gradient of functional (10). We start from $D^{(0)}=\left[\begin{array}{llll}60 & 6 & 15 / 4 & 27 / 10\end{array}\right]^{T}$ and after twelve convergent Newton iterations (11) we obtain the following limiting set of parameters: $D^{(12)}=\left[\begin{array}{lllll}41.94743 & 5.49008 & 3.21407 & 2.49168\end{array}\right]^{T}$. Corresponding piecewise constant exponents $p(t)$ for starting iteration $D^{(0)}$ and for limiting iteration $D^{(12)}$ are shown in Fig. 2, $b$. The functional value is $S\left(D^{(12)}\right)=0.00091828623015$ and in the point $x=1$, where moisture leaves the sample, the moisture concentration $w(1, t)$ is a jump function (see Fig. 2,c). Jumps are not acceptable for $t=1 / 20$ and $t=6 / 20$. Discontinuity of $p(t)$ in these points is probably the reason of the discontinuity of $w(1, t)$. So, we will try to make function $p(t)$ more smoother. Figure 3, $a$ shows besides limiting piecewise constant exponent (solid line) also two other exponents - Fermi exponent (dashed line) and convex exponent (dotted line) of the following analytical form:

$p(t)=p_{1}+\frac{p_{2}-p_{1}}{1+\left(p_{2} / p_{1}-1\right) \exp \left[30\left(t-p_{3} / 20\right)\right]}, \quad p(t)=p_{1}+p_{2}(1-t)^{p_{3}}, \quad 0 \leqslant t \leqslant 1$.

If we start from the initial set of parameters $D^{(0)}=\left[\begin{array}{llll}40 & 2 & 4 & 4\end{array}\right]^{T}$, after twelve convergent Newton iterations we have the following set of limiting parameters for Fermi expo-

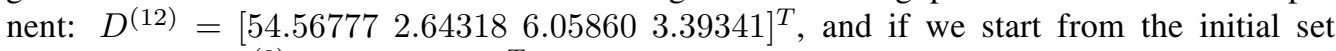
of parameters $D^{(0)}=\left[\begin{array}{llll}60 & 2 & 4 & 6\end{array}\right]^{T}$, we have the following set of parameters for convex exponent: $D^{(12)}=\left[\begin{array}{ll}57.16013 & 2.63424 \\ 4.33721 & 7.66498\end{array}\right]^{T}$. Appropriate functional values are $S\left(D^{(12)}\right)=0.00068812489061$ for Fermi and $S\left(D^{(12)}\right)=0.00067954591778$ for convex exponent. Concentration $w(1, t)$ of moisture that leaves the sample for Fermi exponent (dashed line) and for convex exponent (solid line) can be seen in Fig. 3, $b$. A convex behavior of the moisture $w(1, t)$ is expected when isothermal distribution inside the sample is considered during the experiment of the sample drying. Finally, in Fig. 3, $c$ we compare calculated and experimental moisture profiles for the case of convex exponent $p(t)$ that are fitted quite well.
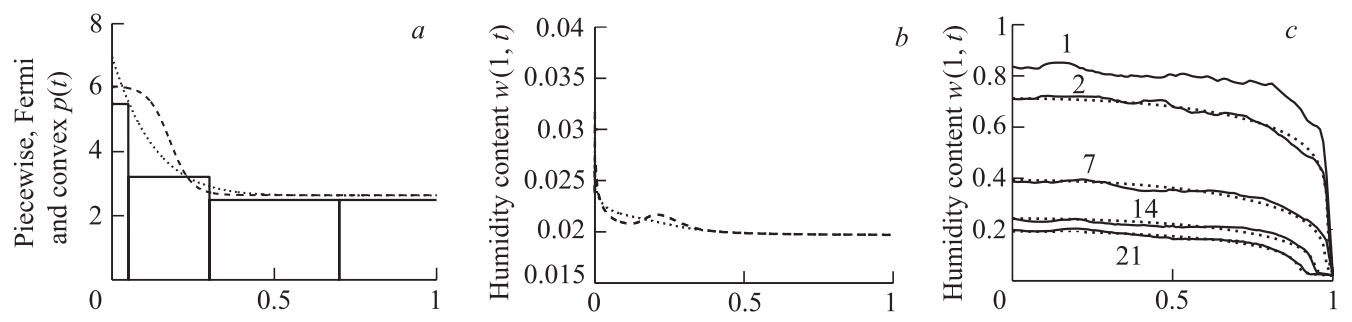

Fig. 3. a) Three limiting exponents $p(t)$ : piecewise constant exponent (solid line), Fermi exponent (dashed line) and convex exponent (dotted line). b) Moisture concentration $w(1, t)$ at the outlet of the sample for Fermi exponent (dashed line) and convex exponent (dotted line). c) Experimental (solid lines) and calculated (dotted lines) profiles in the case of convex exponent $p(t)$, where functional value is $S\left(D^{(12)}\right)=0.00067954591778$ 


\section{ALGORITHM OF THE METHOD}

The following algorithm can be suggested for determination of transfer coefficient as a result of previous consideration:

1) set the term of type $1.5 \exp \left[-150\left(w-v_{0}\right)\right]$ by solving an appropriate direct problem;

2) look for a piecewise exponent $p(t)$ by solving the set of initial-boundary value problems (starting from a measured profile we calculate a new moisture profile that is close to the next measured profile);

3) specify the starting piecewise exponent $p(t)$ by means of convergent Newton method and obtain a limit piecewise exponent $p(t)$;

4) study physical characteristics for the limit exponent $p(t)$ like moisture content $w(1, t)$, flux $-D(1, t) \partial w(1, t) / \partial x$ or other one;

5 ) if characteristics are sufficient, then the final $p(t)$ is found as well as the appropriate transfer coefficient, so go to the point 9;

6) if characteristics are not sufficient, then improve the exponent $p(t)$ (to make it more smoother, convex or so);

7) specify the new suggested exponent $p(t)$ by means of convergent Newton method;

8) go to point 4 ;

9) end.

\section{CONCLUSION}

The limit piecewise exponent (Fig. 2,b) leads us to non-physical solution (Fig. 2,c); however, the exponent is important because the Fermi and convex exponents (Fig. 3, a) are constructed as an approximation of the limit piecewise exponent. The Fermi exponent provides a solution (Fig. 3, b, dashed line) which can be treated as a physical one when the real temperature of the sample (which is not included in considered model) is changed. This solution was obtained in [3]. If it is supposed that the temperature of the sample is constant during the experiment, then we expect the convex solution (Fig. 3, $b$, dotted line) because the exponent $p(t)$ is convex.

Acknowledgements. We thank Mgr. Andrea Matejčíková for English improvement of the paper.

\section{REFERENCES}

1. Pleinert H., Sadouki H., Wittmann F. H. // Materials and Structures. 1998. V.31. P. 218-224.

2. Amirkhanov I. V. et al. // Bull. of PFUR. Ser. «Appl. Comp. Math.». 2005. V.4, No. 1. P. 82-92.

3. Amirkhanov I. V. et al. JINR Preprint P11-2005-158. Dubna, 2005. P. 12 (in Russian). 